\title{
Primary Hyperparathyroidism Misdiagnosed as Giant Cell Bone Tumor of Maxillary Sinus: A Case Report
}

\author{
Leila Aghaghazvini, ${ }^{1,2, *}$ Hashem Sharifian, ${ }^{2,3}$ and Bahman Rasuli ${ }^{2}$ \\ ${ }^{1}$ Department of Radiology, Shariati Hospital, Tehran University of Medical Sciences, Tehran, Iran \\ ${ }^{2}$ Advanced Diagnostic and Interventional Radiology Research Center (ADIR), Tehran University of Medical Sciences, Tehran, Iran \\ ${ }^{3}$ Department of Radiology, Amiralam Hospital, Tehran University of Medical Sciences, Tehran, Iran

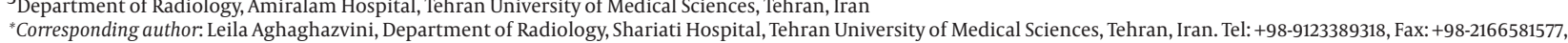 \\ E-mail: la_ghazvini@yahoo.com
}

Received 2013 July 21; Accepted 2014 March 17.

\begin{abstract}
Primary hyperparathyroidism is an endocrine disorder recognized by hyperfunction of parathyroid gland, which can result in persistent bone absorption and brown tumor. Facial involvement of brown tumor is rare and usually involves the mandible. Giant cell tumor ( GCT) is an expansile osteolytic bone tumor which is very similar in clinical, radiological and histological features to brown tumor. Herein, we present a 35-year-old woman with an 11-month history of gradually swelling of the right maxilla and buccal spaces began during pregnancy two years ago. No other clinical or laboratory problems were detected. Postpartum CT scan demonstrated a lytic expansile multi-septated mass lesion containing enhancing areas, which initially described as GCT of the right maxillary sinus following surgery. Four months later, gradual progressive swelling of the bed of tumor was recurred and revised pathological slices were compatible with GCT. Regarding patient recent paresthesia, repeated laboratory tests were performed. Finally, according to laboratory results (elevation of serum calcium and parathyroid hormone), ultrasonographic findings and radioisotope scan (Sestamibi), probable parathyroid mass and brown tumor of maxilla was diagnosed. Pathology confirmed hyperplasia of right inferior parathyroid gland. Our case was thought-provoking due to its interesting clinical presentation and unusual presentation of brown tumor in parathyroid hyperplasia.
\end{abstract}

Keywords: Brown Tumor, Hyperparathyroidism, Giant Cell Tumor, Maxilla

\section{Introduction}

Brown tumor is an unusual bone change created by primary hyperparathyroidism affected less than $2 \%$ of primary hyperparathyroidism patients (1-3). Brown tumors have no neoplastic behavior but are a reparative cellular response.

Histological feature of brown tumor is delineated by soft tissue swelling consisted of hemorrhage foci, cystic like areas lining with connective contexture and fibrovascular tissue surrounding multinucleated cells namely giant cell (3). Patients with parathyroid gland dysfunction classified to subtypes of pathological disturbance (adenoma, hyperplasia, secondary type of hyperparathyroidism and para-neoplastic syndromes) recognized by elevated levels of serum calcium and parathyroid hormone on routine laboratory findings. Brown tumor; rapid turnover of osteoclastic cells; results from hypersecretion of parathyroid hormone (PTH) for a long time (4). Usually advanced lytic lesions such as brown tumor are accompanied by symptoms produced by hypercalcemia. Brown tumor is recognized only in less than $5 \%$ of patients with hyperparathyroidism. Flank pain produced by renal stones, gastrointestinal tract disorders and neurologi- cal disturbances are other symptoms of hypercalcemia $(5,6)$. Common structures affected by brown tumor are bony thorax (ribs and clavicle), pelvic rim and long bones (tibia and femur). Involvement of maxilla is rare; furthermore, maxillary brown tumor is unusual and mostly accompanied by other brown tumors of facial bones such as mandible and hard palate (7).

Our patient had a lytic lesion (brown tumor) in right maxillary sinus produced by atypical hyperplasia of parathyroid gland. Swelling of right buccal space and maxillary bone due to brown tumor was the first manifestation of primary hyperparathyroidism.

\section{Case Presentation}

A 35-year-old woman was referred with an 11-month history of gradually swelling of the right maxilla and buccal spaces began during pregnancy two years ago. Patient felt no facial pain or paresthesia.

There was no history of trauma to the facial bones, nasal obstruction, dental problem, epistaxis, drugs consumption or visual change.

Copyright (C) 2016, Tehran University of Medical Sciences and Iranian Society of Radiology. This is an open-access article distributed under the terms of the Creative Commons Attribution-NonCommercial 4.0 International License (http://creativecommons.org/licenses/by-nc/4.0/) which permits copy and redistribute the material just in noncommercial usages, provided the original work is properly cited. 
On physical examination, facial asymmetry was found due to swelling of right maxilla without significant tenderness.

No other clinical or laboratory problems were found. Postpartum CT scan of the maxillofacial region revealed a large lytic expansile multi-septated mass along the right maxillary sinus and buccal region $(60 \times 52 \times 45 \mathrm{~mm})$ (Figure 1 ). In this patient, surgical excision of maxillary lesion was performed and histopathology was compatible with a giant cell tumor (GCT) of bone. Biopsy revealed a benign process consisting of significant number of osteoclasts with little osteoblast cells and small component of fibrosis and osteoid formation without evidence of malignant features.
Four months following surgery, gradual progressive swelling of the bed of tumor was recurred and revised pathological slices were compatible with GCT. Regarding patient recent paresthesia, repeated laboratory tests were performed. According to laboratory tests (elevation of serum calcium and parathyroid hormone), ultrasonography and radioisotope scan (Sestamibi) were considered. In ultrasonography, a $11 \times 14 \times 27 \mathrm{~mm}$ mixed hypo echo mass lesion was seen in posterior aspect of right thyroid lobe compatible with probable vascular parathyroid mass (Figure 2). In sestamibi isotope scan, increased uptake in posterior aspect of right thyroid lobe suggested a right parathyroid adenoma or hyperplasia (Figure 3). Other bone survey was unremarkable.
A

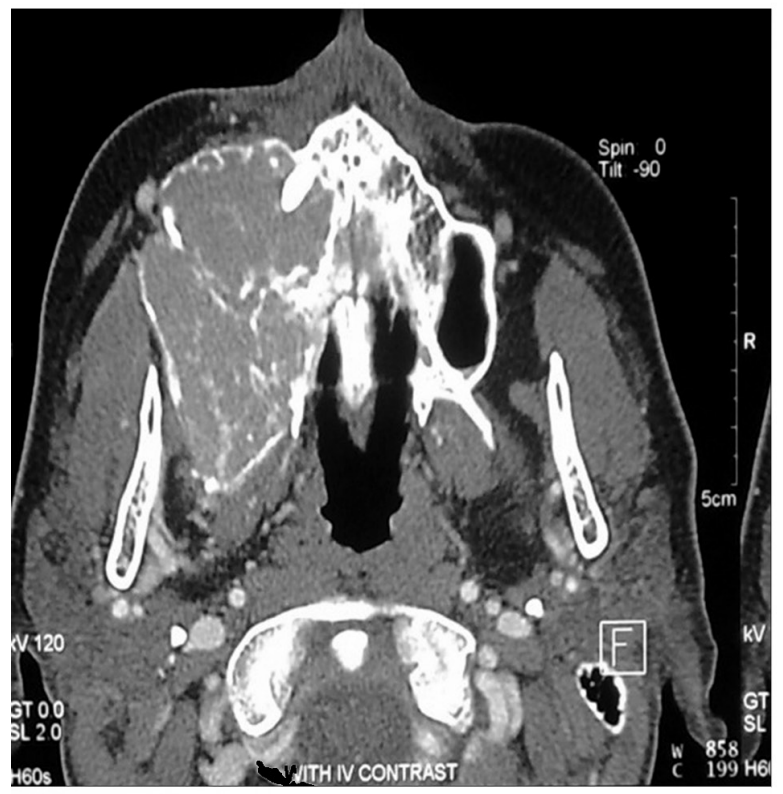

B

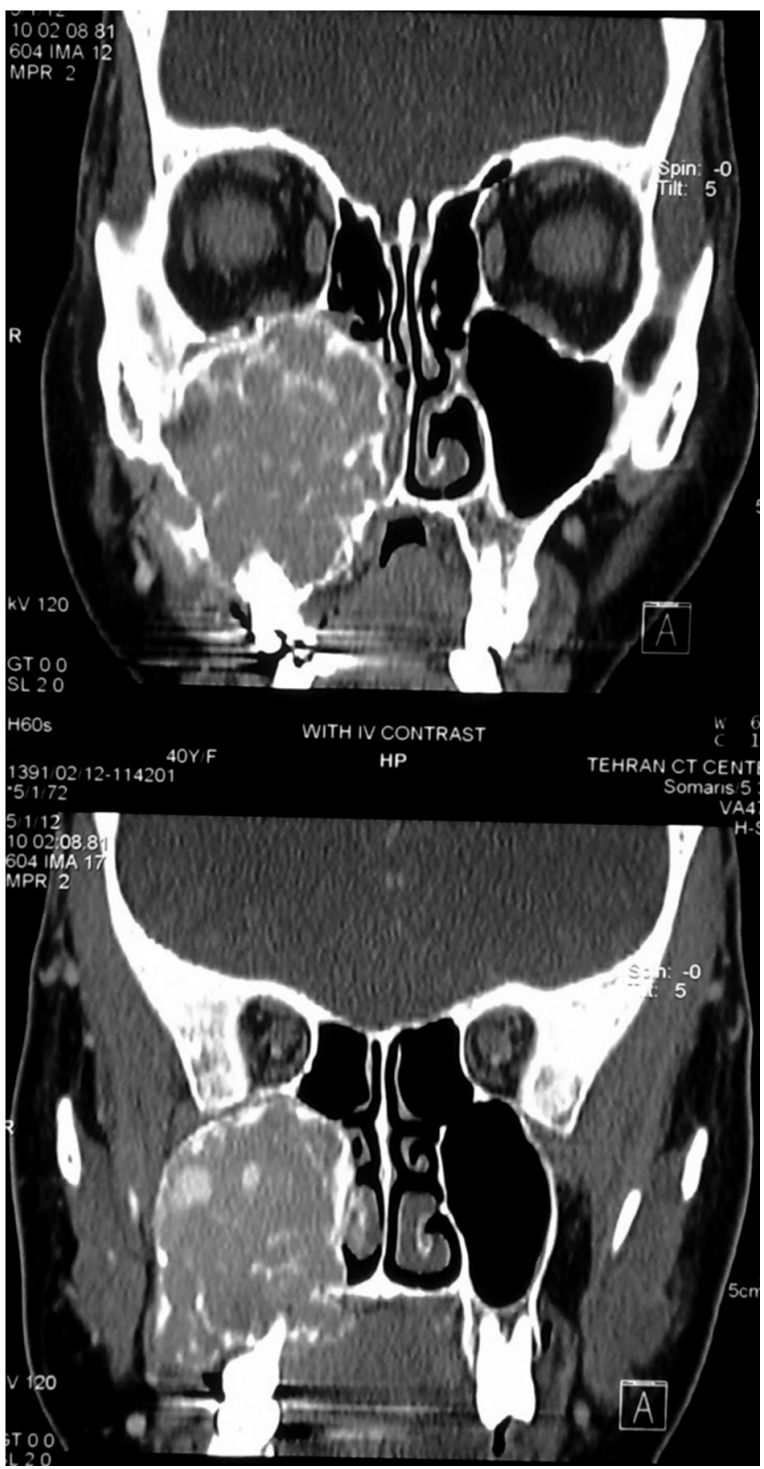

Figure 1. A 35-year-old woman with gradually swelling of the right maxilla. Axial (A) and coronal (B) view of postcontrast facial CT revealed a large lytic expansile multi-septated mass lesion in right maxillary sinus containing septa. Adjacent soft tissue is unremarkable. 


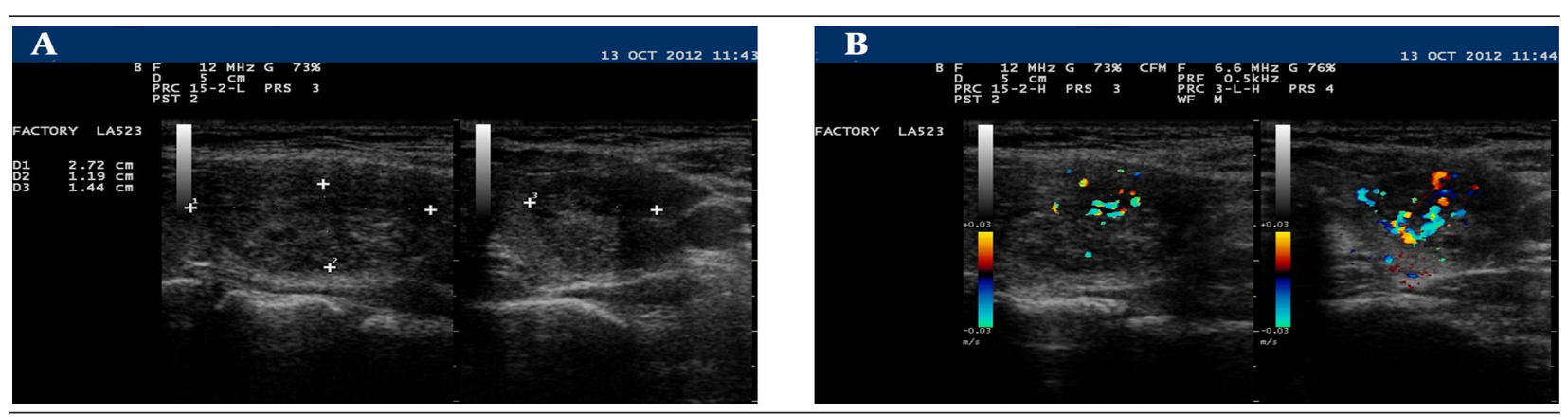

Figure 2. Neck sonography showed a $27 \times 14 \times 11 \mathrm{~mm}$ mixed hypo echoic mass lesion in posterior aspect of right thyroid lobe compatible with vascular parathyroid mass $(\mathrm{A}, \mathrm{B})$.

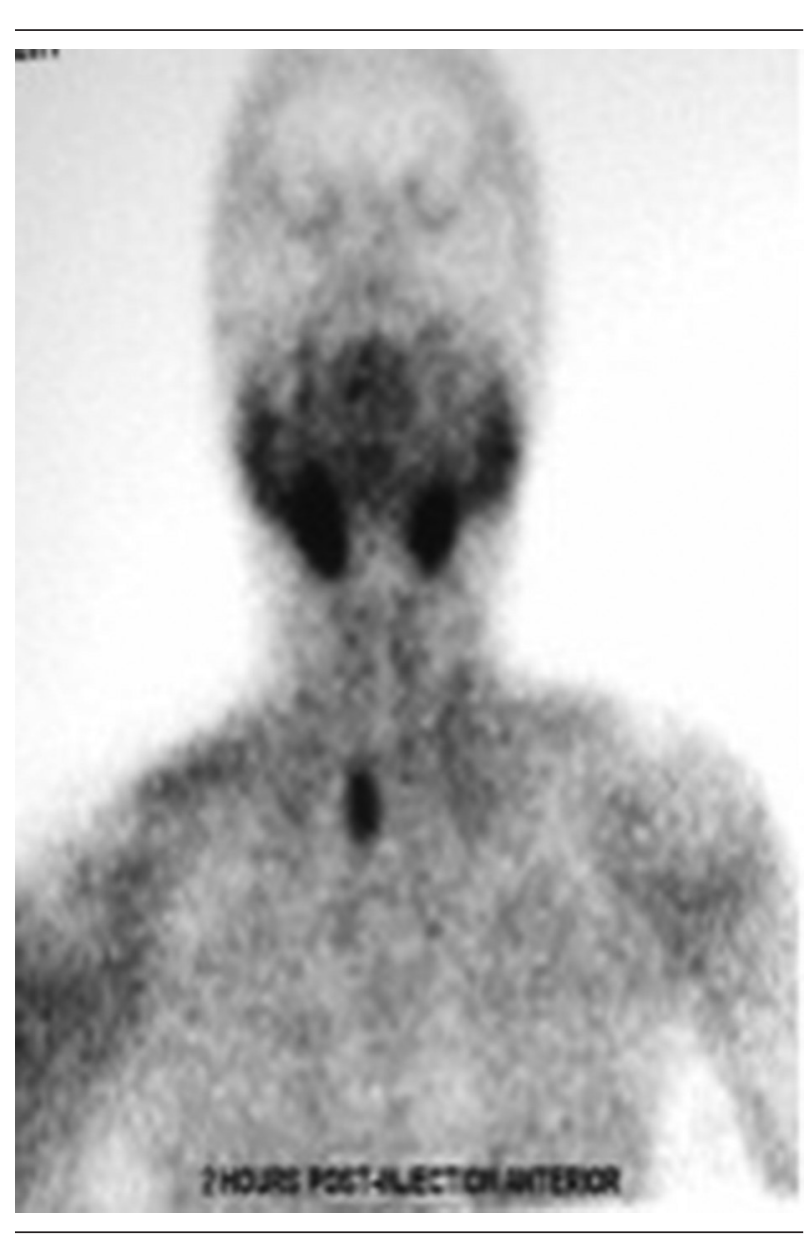

Figure 3. Radioisotope scan (Tc-99 m sestamibi) depicted a right parathyroid hyperplasia. Persistent concentration of radiotracer (after 2 hours injection) in posterior aspect of right thyroid lobe by Tc $99 \mathrm{~m}$ sestamibi scan delineated a right parathyroid hyperplasia.

After diagnosis of parathyroid mass, surgical excision was performed. Pathological findings of the right parathyroid gland revealed an encapsulated mass with solid-cystic component. Microscopically, the mass had a nodular growth pattern. Cells organizing the mass had a uniform shape with rounded nucleoli and pale eosinophilic cytoplasm without evidence of fat within the cytoplasm (Figure 4). Pathological and surgical findings were compatible and consistent with parathyroid hyperplasia.
Diagnosis of brown tumor due to primary hyperparathyroidism related to parathyroid hyperplasia was achieved according to clinical and pathological findings.

\section{Discussion}

Hyperparathyroidism is an endocrine disorder characterized by elevation of parathyroid hormone levels. The most common causes of primary hyperparathyroidism (PHP) are adenoma (81\%), hyperplasia (15\%) and carcinoma $(0.5 \%-4 \%)$ of parathyroid gland $(1,2)$.

PHP is most often occurred in patients older than 50 years with females preponderant. At the time of diagnosis of primary hyperparathyroidism (PHP), merely $2 \%$ of patients are younger than 30 years (4). Primary and secondary hyperparathyroidism had similar clinical presentations, but the primary type is more severe than the secondary type $(2,3)$.

Brown tumors are consequence of an imbalance between osteoclast and osteoblast cells activity, which can lead to bone resorption and its substitution by fibrous tissue (4).

Unlike the term, brown tumor is misnomer, because they are not neoplastic lesions. Substitutes for the term of brown tumor have been recommended, but not accepted yet.

Nowadays, diagnosis of brown tumor in asymptomatic patients is incidental detection of high serum level of calcium (1-7).

But yet in some patients, the only late presentation of brown tumor is bony manifestations.

Involvement of facial bones by the brown tumor is extremely rare, especially in the maxilla and hard palate (7).

On imaging, bony manifestations of brown tumor are a lytic well defined border lesion along with bone expansion and cortical thinning. Generalized osteopenia may also be seen.

Histologically differentiation between Brown tumors and other giant cell-containing lesions such as aneurysmal bone cyst (ABC), GCT and giant cell consisting granuloma is indistinguishable (5-7).

The important aspect of our case was maxillary brown tumor as the initial presentation of an atypical parathyroid hyperplasia without involvement of mandible, palate and other facial bones, which at first misdiagnosed as GCT.

Therefore, screening both hypercalcemia and hyperparathyroidism in all patients referred with a recurrent GCT in facial bones should be performed. 

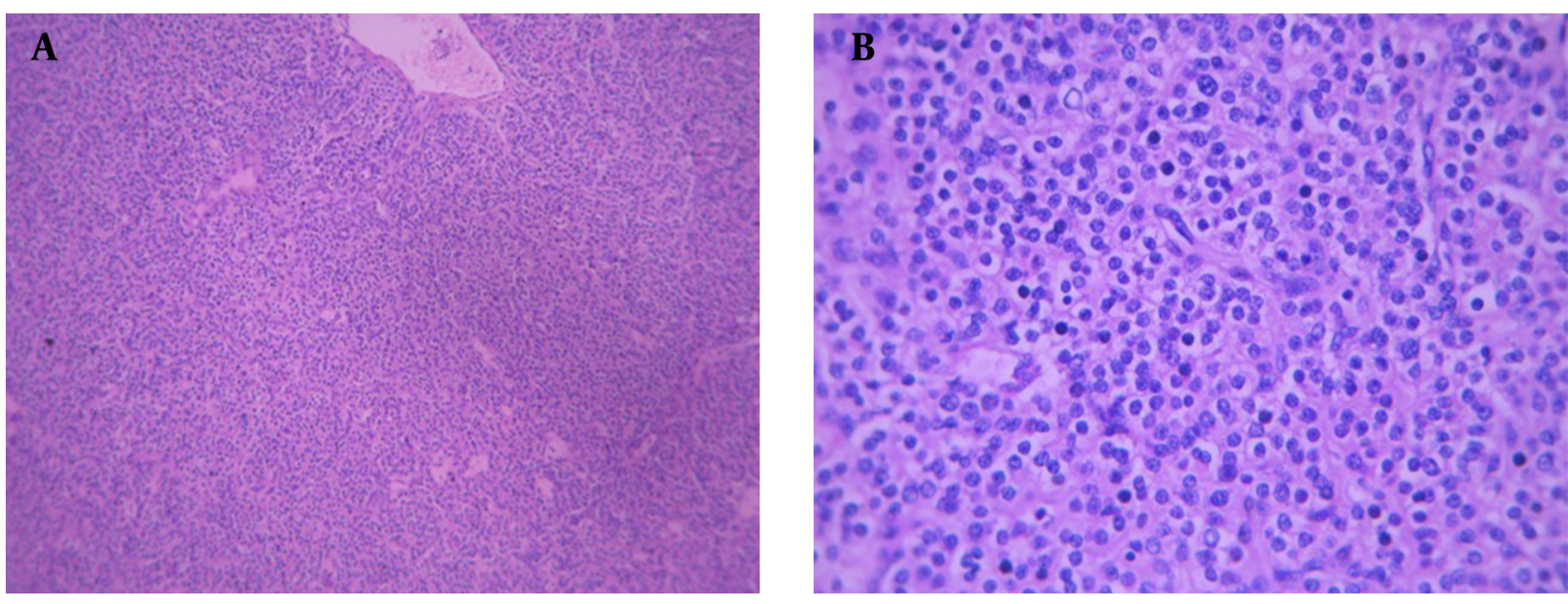

Figure 4. In histopathology, the cells had a uniform shape with rounded nucleoli and pale eosinophilic cytoplasm without evidence of fat within the cytoplasm. (A) $\mathrm{H}$ and $\mathrm{E}(\times 40)$, (B) $\mathrm{H}$ and $\mathrm{E}(\times 100)$

Finally, excision of parathyroid tumor in our patient was performed and biopsy revealed histologic features of parathyroid hyperplasia.

After excision of parathyroid gland tumor and decreased levels of biochemical parameters, partial or completely regression of brown tumors occurs spontaneously (7).

Indication for surgical resection of brown tumors is a deformed and large lesion or weakening of affected bone. Although, other authors (7) believe that surgical excision of residual brown tumors should be performed after initial treatment with systemic corticosteroids to decrease the size of tumors.

In our case, also no surgical excision was considered after diagnosis of parathyroid hyperplasia; the brown tumor disappeared after resection of enlarged parathyroid gland.

This patient was interesting because of unusual clinical presentation, unusual occurrence of brown tumor in maxilla and rare presentation of brown tumor due to parathyroid hyperplasia. This case highlights the importance of thorough diagnostic work-up for all lesions in the maxilla to differentiate recurrent GCT and brown tumors.

\section{Footnote}

Authors' Contribution:Leila Aghaghazvini developed the idea and protocol, abstracted and gathering data, wrote and edited the manuscript, and is guarantor. Hashem Sharifian and Bahman Rasuli contributed to development, abstracted data and prepared and wrote the manuscript.

\section{References}

1. Al-Gahtany M, Cusimano M, Singer W, Bilbao J, Kovacs K, Marotta T. Brown tumors of the skull base. Case report and review of the literature. J Neurosurg. 2003;98(2):417-20.

2. Emin AH, Suoglu Y, Demir D, Karatay MC. Normocalcemic hyperparathyroidism presented with mandibular brown tumor: report of a case. Auris Nasus Larynx. 2004;31(3):299-304.

3. Daniels JSM. Primary hyperparathyroidism presenting as a palatal brown tumor. Oral Surg, Oral Med, Oral Pathol, Oral Radiol, and Endodontol. 2004;98(4):409-13.

4. Resendiz-Colosia JA, Rodriguez-Cuevas SA, Flores-Diaz R, Juan $\mathrm{MH}$, Gallegos-Hernandez JF, Barroso-Bravo S, et al. Evolution of maxillofacial brown tumors after parathyroidectomy in primary hyperparathyroidism. Head Neck. 2008;30(11):1497-504.

5. Sutbeyaz Y, Yoruk O, Bilen H, Gursan N. Primary hyperparathyroidism presenting as a palatal and mandibular brown tumor.J Craniofac Surg. 2009;20(6):2101-4

6. Kar DK, Gupta SK, Agarwal A, Mishra SK. Brown tumor of the palate and mandible in association with primary hyperparathyroidism. J Oral Maxillofac Surg. 2001;59(11):1352-4.

7. Martinez-Gavidia EM, Bagan JV, Milian-Masanet MA, Lloria de Miguel E, Perez-Valles A. Highly aggressive brown tumour of the maxilla as first manifestation of primary hyperparathyroidism. Int J Oral Maxillofac Surg. 2000;29(6):447-9. 